\title{
An Overview of Scalable FETI-DP Algorithms for Variational Inequalities
}

\author{
Zdeněk Dostál ${ }^{1}$, David Horák ${ }^{1}$ and Dan Stefanica ${ }^{2}$ \\ 1 FEI VŠB-Technical University Ostrava, CZ-70833 Ostrava, Czech Republic \\ zdenek.dostal@vsb.cz, david.horak@vsb.cz \\ 2 Baruch College, City University of New York, NY 10010, USA \\ Dan_Stefanica@baruch . cuny.edu
}

Summary. We review our recent results concerning optimal algorithms for numerical solution of both coercive and semi-coercive variational inequalities by combining dual-primal FETI algorithms with recent results for bound and equality constrained quadratic programming problems. The convergence bounds that guarantee the scalability of the algorithms are presented. These results are confirmed by numerical experiments.

\section{Introduction}

The Finite Element Tearing and Interconnecting (FETI) method was originally proposed by Farhat and Roux [14] as a parallel solver for problems described by elliptic partial differential equations. After introducing a socalled "natural coarse grid", Farhat, Mandel and Roux [13] modified the basic FETI method to obtain a numerically scalable algorithm. A similar result was achieved by the Dual-Primal FETI method (FETI-DP) introduced by Farhat et al. [12]; see also [15]. In this paper, we use the FETI-DP method to develop scalable algorithms for the numerical solution of elliptic variational inequalities. The FETI-DP methodology is first applied to the variational inequality to obtain either a strictly convex quadratic programming problem with non-negativity constraints, or a convex quadratic programming problem with bound and equality constraints. This problems are then solved efficiently by recently proposed improvements $[4,11]$ of the active set based proportioning algorithm [3], possibly combined with a semimonotonic augmented Lagrangian algorithm $[5,6]$. The rate of convergence of these algorithms can be bounded in terms of the spectral condition number of the quadratic problem, and therefore the scalability of the resulting algorithm can be established provided that suitable bounds on the condition number of the Hessian of the quadratic cost function exist. We present such estimates in terms of the decomposition parameter $H$ and the discretization parameter $h$. These bounds are independent 
of both the decomposition of the computational domain and the discretization, provided that we keep the ratio $H / h$ fixed. We report numerical results that are in agreement with the theory and confirm the numerical scalability of our algorithm. Let us recall that an algorithm based on FETI-DP and on active set strategies with additional planning steps, FETI-C, was introduced by Farhat et al. [1]. The scalability of FETI-C was established experimentally.

\section{Model problem}

To simplify our exposition, we restrict our attention to a simple model problem. The computational domain is $\Omega=\Omega^{1} \cup \Omega^{2}$, where $\Omega^{1}=(0,1) \times(0,1)$ and $\Omega^{2}=(1,2) \times(0,1)$, with boundaries $\Gamma^{1}$ and $\Gamma^{2}$, respectively. We denote by $\Gamma_{u}^{i}, \Gamma_{f}^{i}$, and $\Gamma_{c}^{i}$ the fixed, free, and potential contact parts of $\Gamma^{i}, i=1,2$. We assume that $\Gamma_{u}^{1}$ has non-zero measure, i.e., $\Gamma_{u}^{1} \neq \emptyset$. For a coercive model problem, $\Gamma_{u}^{2} \neq \emptyset$, while for a semicoercive model problem, $\Gamma_{u}^{2}=\emptyset$; see Figure 1a. Let $\Gamma_{c}=\Gamma_{c}^{1} \cup \Gamma_{c}^{2}$. The Sobolev space of the first order on $\Omega^{i}$ is denoted by $H^{1}\left(\Omega^{i}\right)$ and the space of Lebesgue square integrable functions is denoted by $L^{2}\left(\Omega^{i}\right)$. Let $V=V^{1} \times V^{2}$, with

$$
V^{i}=\left\{v^{i} \in H^{1}\left(\Omega^{i}\right): v^{i}=0 \quad \text { on } \quad \Gamma_{u}^{i}\right\}, \quad i=1,2 .
$$

Let $\mathcal{K} \subset V$ be a closed convex subset of $\mathcal{H}=H^{1}\left(\Omega^{1}\right) \times H^{1}\left(\Omega^{2}\right)$ defined by

$$
\mathcal{K}=\left\{\left(v^{1}, v^{2}\right) \in V: v^{2}-v^{1} \geq 0 \quad \text { on } \quad \Gamma_{c}\right\} .
$$

We define the symmetric bilinear form $a(\cdot, \cdot): \mathcal{H} \times \mathcal{H} \rightarrow R$ by

$$
a(u, v)=\sum_{i=1}^{2} \int_{\Omega^{i}}\left(\frac{\partial u^{i}}{\partial x_{1}} \frac{\partial v^{i}}{\partial x_{1}}+\frac{\partial u^{i}}{\partial x_{2}} \frac{\partial v^{i}}{\partial x_{2}}\right) d x .
$$

Let $f \in L^{2}(\Omega)$ be a given function and $f^{i} \in L^{2}\left(\Omega^{i}\right), i=1,2$, be the restrictions of $f$ to $\Omega^{i}, i=1,2$. We define the linear form $l(\cdot): \mathcal{H} \rightarrow R$ by

$$
\ell(v)=\sum_{i=1}^{2} \int_{\Omega^{i}} f^{i} v^{i} d x
$$

and consider the following problem:

$$
\text { Find } \min \frac{1}{2} a(u, u)-\ell(u) \text { subject to } u \in \mathcal{K} \text {. }
$$

The solution of the model problem may be interpreted as the displacement of two membranes under the traction $f$. The left membrane $\Omega^{1}$ is fixed on the left edge as in Figure 1a and the left edge of $\Omega^{2}$ is not allowed to penetrate below the right edge of $\Omega^{1}$. For the model problem to be well defined, we 


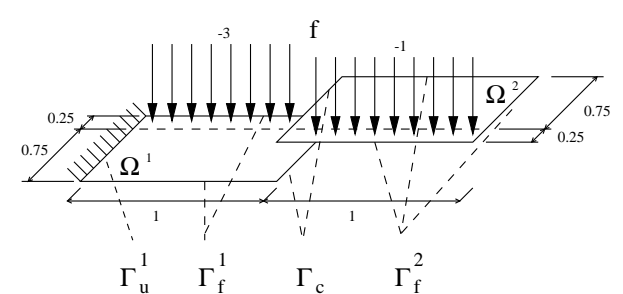

Fig. 1a: Semi-coercive model problem

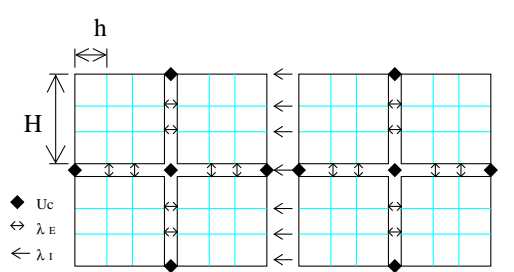

Fig. 1b: Decomposition: $H=.5, H / h=3$

either require that the right edge of the right membrane $\Omega^{2}$ is fixed, for the coercive problem, or, for the semicoercive problem, that the traction function $f$ satisfies

$$
\int_{\Omega^{2}} f d x<0
$$

\section{FETI-DP discretization of the problem}

The first step in our domain decomposition method is to partition each domain $\Omega^{i}, i=1,2$, on a rectangular grid into subdomains of diameter of order $H$. Let $W$ be the finite element space whose restrictions to $\Omega^{1}$ and $\Omega^{2}$ are $Q_{1}$ finite element spaces of comparable mesh sizes of order $h$, corresponding to the subdomain grids in $\Omega^{1}$ and $\Omega^{2}$. We call a crosspoint either a corner that belongs to four subdomains, or a corner that belongs to two subdomains and is located on $\partial \Omega^{1} \backslash \Gamma_{u}^{1}$ or on $\partial \Omega^{2} \backslash \Gamma_{u}^{2}$. The nodes corresponding to the end points of $\Gamma_{c}$ are not crosspoints; see Figure 1b. An important feature for developing FETI-DP type algorithms is that a single degree of freedom is considered at each crosspoint, while two degrees of freedom are introduced at all the other matching nodes across subdomain edges. Let $v \in W$. The continuity of $v$ in $\Omega^{1}$ and $\Omega^{2}$ is enforced at every interface node that is not a crosspoint. For simplicity, we also denote by $v$ the nodal values vector of $v \in W$. The discretized version of problem (1) with the auxiliary domain decomposition has the form

$$
\min \frac{1}{2} v^{T} K v-v^{T} f \quad \text { subject to } \quad B_{I} v \leq 0 \quad \text { and } \quad B_{E} v=0
$$

where the full rank matrices $B_{I}$ and $B_{E}$ describe the non-penetration (inequality) conditions and the gluing (equality) conditions, respectively, and $f$ represents the discrete analog of the linear form $\ell(\cdot)$. In $(2), K=\operatorname{diag}\left(K_{1}, K_{2}\right)$ is the block diagonal stiffness matrix corresponding to the model problem (1). The block $K_{1}$ corresponding to $\Omega^{1}$ is nonsingular, due to the Dirichlet boundary conditions on $\Gamma_{u}^{1}$. The block $K_{2}$ corresponding to $\Omega^{2}$ is nonsingular for a coercive problem, and is singular, with the kernel made of a vector $e$ with all 
entries equal to 1 , for a semicoercive problem. The kernel of $K$ is spanned by the matrix $R$ defined by

$$
R=\left[\begin{array}{l}
0 \\
e
\end{array}\right]
$$

Even though $R$ is a column vector for our model problem, we will regard $R$ as a matrix whose columns span the kernel of $K$. We partition the nodal values of $v \in W$ into crosspoint nodal values, denoted by $v_{c}$, and remainder nodal values, denoted by $v_{r}$. The continuity of $v$ at crosspoints is enforced by using a global vector of degrees of freedom $v_{c}^{g}$ and a global-to-local map $L_{c}$ with one nonzero entry equal to 1 in each row, i.e., we require that $v_{c}=L_{c} v_{c}^{g}$. Therefore,

$$
v=\left[\begin{array}{c}
v_{r} \\
v_{c}
\end{array}\right]=\left[\begin{array}{c}
v_{r} \\
L_{c} v_{c}^{g}
\end{array}\right] .
$$

Let $f_{c}$ and $f_{r}$ be the parts of the right hand side $f$ corresponding to the corner and remainder nodes, respectively. Let $B_{I, r}$ and $B_{I, c}$ be the matrices made of the columns of $B_{I}$ corresponding to $v_{r}$ and $v_{c}$, respectively; define $B_{E, r}$ and $B_{E, c}$ similarly. Let

$$
B_{r}=\left[\begin{array}{c}
B_{I, r} \\
B_{E, r}
\end{array}\right], \quad B_{c}=\left[\begin{array}{c}
B_{I, c} \\
B_{E, c}
\end{array}\right], \quad B=\left[\begin{array}{ll}
B_{r} & B_{c}
\end{array}\right] .
$$

Let $K_{r r}, K_{r c}$, and $K_{c c}$ denote the blocks of $K$ corresponding to the decomposition of $v$ into $v_{r}$ and $v_{c}$. Consider the shortened vectors

$$
\bar{v}=\left[\begin{array}{l}
v_{r} \\
v_{c}^{g}
\end{array}\right] \in \bar{W}
$$

Let $\lambda_{I}$ and $\lambda_{E}$ be Lagrange multipliers enforcing the inequality and redundancy conditions. The Lagrangian $L(v, \lambda)=1 / 2 v^{T} K v-v^{T} f+v^{T} B^{T} \lambda$ associated with problem (2) can be expressed as follows:

$$
L(\bar{v}, \lambda)=\frac{1}{2} \bar{v}^{T} \bar{K} \bar{v}-\bar{v}^{T} \bar{f}+\bar{v}^{T} \bar{B}^{T} \lambda,
$$

where

$$
\lambda=\left[\begin{array}{c}
\lambda_{I} \\
\lambda_{E}
\end{array}\right], \quad \bar{K}=\left[\begin{array}{ll}
K_{r r} & K_{r c} L_{c} \\
L_{c}^{T} K_{r c}^{T} & L_{c}^{T} K_{c c} L_{c}
\end{array}\right], \quad \bar{B}=\left[\begin{array}{ll}
B_{r} & B_{c} L_{c}
\end{array}\right], \quad \bar{f}=\left[\begin{array}{r}
f_{r} \\
L_{c}^{T} f_{c}
\end{array}\right] .
$$

Using duality theory [2], we can eliminate the primal variables $v$ from the mixed formulation of (2). For a coercive problem, $K$ is nonsingular and we obtain the problem of finding

$$
\min \Theta(\lambda)=\min \frac{1}{2} \lambda^{T} F \lambda-\lambda^{T} \tilde{d} \quad \text { s.t. } \quad \lambda_{I} \geq 0,
$$

with $F=\bar{B} \bar{K}^{-1} \bar{B}^{T}$ and $\widetilde{d}=\bar{B} \bar{K}^{-1} \bar{f}$. For an efficient implementation of $F$ it is important to exploit the structure of $K$; see $[8,10]$ for more details. 
For a semicoercive problem, we obtain the problem of finding

$$
\min \Theta(\lambda)=\min \frac{1}{2} \lambda^{T} F \lambda-\lambda^{T} \widetilde{d} \quad \text { s.t. } \quad \lambda_{I} \geq 0 \quad \text { and } \quad \widetilde{G} \lambda=\widetilde{e},
$$

where $F=\bar{B} \bar{K}^{\dagger} \bar{B}^{T}, \widetilde{d}=\bar{B} \bar{K}^{\dagger} \bar{f}, \widetilde{G}=R^{T} \bar{B}^{T}, \widetilde{e}=R^{T} \bar{f}$. Here, $\bar{K}^{\dagger}$ denotes a suitable generalized inverse that satisfies $\bar{K} \bar{K}^{\dagger} \bar{K}=\bar{K}$. Even though problem (5) is much more suitable for computations than (1) and was used for solving discretized variational inequalities efficiently [7], further improvement may be achieved as follows. Let $\widetilde{T}$ denote a nonsingular matrix that defines the orthonormalization of the rows of $\widetilde{G}$ such that the matrix $G=\widetilde{T} \widetilde{G}$ has orthonormal rows. Let $e=\widetilde{T} \widetilde{e}$. Then, problem (5) reads

$$
\min \frac{1}{2} \lambda^{T} F \lambda-\lambda^{T} \widetilde{d} \quad \text { s.t } \quad \lambda_{I} \geq 0 \quad \text { and } \quad G \lambda=e .
$$

Next, we transform the problem of minimization on the subset of the affine space to a minimization problem on the subset of a vector space. Let $\widetilde{\lambda}$ be an arbitrary feasible vector such that $G \widetilde{\lambda}=e$. We look for the solution $\lambda$ of (5) in the form $\lambda=\mu+\widetilde{\lambda}$. After returning to the old notation by replacing $\mu$ by $\lambda$, it is easy to see that (6) is equivalent to

$$
\min \frac{1}{2} \lambda^{T} F \lambda-d^{T} \lambda \quad \text { s.t } \quad G \lambda=0 \quad \text { and } \quad \lambda_{I} \geq-\widetilde{\lambda_{I}},
$$

with $d=\widetilde{d}-F \tilde{\lambda}$. Our final step is based on the observation that the augmented Lagrangian for problem (7) may be decomposed by the orthogonal projectors

$$
Q=G^{T} G \quad \text { and } \quad P=I-Q
$$

on the image space of $G^{T}$ and on the kernel of $G$, respectively. Since $P \lambda=\lambda$ for any feasible $\lambda$, problem (7) is equivalent to

$$
\min \frac{1}{2} \lambda^{T} P F P \lambda-\lambda^{T} P d \quad \text { s.t } \quad G \lambda=0 \quad \text { and } \quad \lambda_{I} \geq-\widetilde{\lambda_{I}} .
$$

\section{Optimality}

To solve the discretized variational inequality, we use our recently proposed algorithms $[8,10]$. To solve the bound constrained quadratic programming problem (4), we use active set based algorithms with proportioning and gradient projections $[4,11]$. The rate of convergence of the resulting algorithm can be estimated in terms of bounds on the spectrum of the Hessian of $\Theta$. To solve the bound and equality constrained quadratic programming problem (8), we use semimonotonic augmented Lagrangian algorithms $[5,6]$. The equality constraints are enforced by Lagrange multipliers generated in the 
outer loop, while the bound constrained problems are solved in the inner loop by the above mentioned algorithms. The rate of convergence of this algorithm may be again described in terms of bounds on the spectrum of the Hessian of $\Theta$. Summing up, the optimality of our algorithms is guaranteed, provided that we establish optimal bounds on the spectrum of the Hessian of $\Theta$. Such bounds on the spectrum of the operator $F$, possibly restricted to $\operatorname{Im} P$, are given in the following theorem:

Theorem 1. If $F$ denotes the Hessian matrix of $\Theta$ in (4), the following spectral bounds hold:

$$
\lambda_{\max }(F)=\|F\| \leq C\left(\frac{H}{h}\right)^{2} ; \quad \lambda_{\min }(F) \geq C .
$$

If $F$ denotes the Hessian matrix of $\Theta$ in (5), the following spectral bounds hold:

$$
\lambda_{\max }(F \mid \operatorname{Im} P) \leq\|F\| \leq C\left(\frac{H}{h}\right)^{2} ; \quad \lambda_{\min }(F \mid \operatorname{Im} P) \geq C .
$$

Proof: See $[8,10]$.

\section{Numerical experiments}

We report some results for the numerical solutions of a coercive contact problem and of a semicoercive contact problem, in order to illustrate the performance and numerical scalability of our FETI-DP algorithms. In our experiments, we used a function $f$ vanishing on $(0,1) \times[0,0.75) \cup(1,2) \times[0.25,1)$. For the coercive problem, $f$ was equal to -1 on $(0,1) \times[0.75,1)$ and to -3 on $(1,2) \times[0,0.25)$, while for the semicoercive problem, $f$ was equal to -5 on $(0,1) \times[0.75,1)$ and to -1 on $(1,2) \times[0,0.25)$. Each domain $\Omega^{i}$ was partitioned into identical squares with side $H=1 / 2,1 / 4,1 / 8$. These squares were then discretized by a regular grid with the stepsize $h$. For each partition, the number of nodes on each edge, $H / h$, was taken to be 4,8 , and 16 . The meshes matched across the interface for every neighboring subdomains. All experiments were performed in MATLAB. The solution of both the coercive and semicoercive model problems for $H=1 / 4$ and $h=1 / 4$ are presented in Figure 2. Selected results of the computations for varying values of $H$ and $H / h$ are given in Table 1 , for the coercive problem, and in Table 2 for the semicoercive problem. The primal dimension/dual dimension/number of corners are recorded in the upper row in each field of the table, while the number of the conjugate gradient iterations required for the convergence of the solution to the given precision is recorded in the lower row. The key point is that the number of the conjugate gradient iterations for a fixed ratio $H / h$ varies very moderately with the increasing number of subdomains. 
Table 1. Convergence results for the FETI-DP algorithm - coercive problem

\begin{tabular}{|c|c|c|c|}
\hline$H$ & $1 / 2$ & $1 / 4$ & $1 / 8$ \\
\hline \hline$H / h=16$ & $2312 / 153 / 10$ & $9248 / 785 / 42$ & $36992 / 3489 / 154$ \\
& 33 & 39 & 43 \\
\hline$H / h=8$ & $648 / 73 / 10$ & $2592 / 369 / 42$ & $10365 / 1633 / 154$ \\
& 20 & 32 & 34 \\
\hline$H / h=4$ & $200 / 33 / 10$ & $800 / 161 / 42$ & $3200 / 705 / 154$ \\
& 19 & 24 & 27 \\
\hline
\end{tabular}

Table 2. Convergence results for the FETI-DP algorithm - semicoercive problem

\begin{tabular}{|c|c|c|c|}
\hline$H$ & $1 / 2$ & $1 / 4$ & $1 / 8$ \\
\hline \hline$H / h=16$ & $2312 / 153 / 10$ & $9248 / 785 / 42$ & $36992 / 3489 / 154$ \\
& 61 & 51 & 53 \\
\hline$H / h=8$ & $648 / 73 / 10$ & $2592 / 369 / 42$ & $10365 / 1633 / 154$ \\
& 38 & 36 & 46 \\
\hline$H / h=4$ & $200 / 33 / 10$ & $800 / 161 / 42$ & $3200 / 705 / 154$ \\
& 29 & 28 & 35 \\
\hline
\end{tabular}
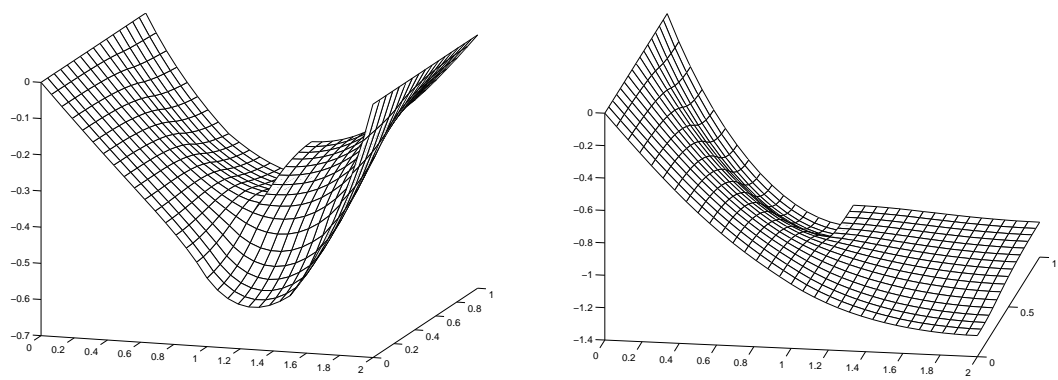

Fig. 2a: Solution of coercive problem Fig. 2b: Solution of semi-coercive problem

\section{Comments and conclusions}

We applied the FETI-DP methodology to the numerical solution of a variational inequality. Theoretical arguments and results of numerical experiments show that the scalability of the FETI-DP method which was established earlier for linear problems may be preserved even in the presence of nonlinear conditions on the contact boundary. The results are supported by numerical experiments. Similar results were obtained also for non-matching contact interfaces discretized by mortars [9].

Acknowledgments. The work of the first two authors was supported by Grant 101/04/1145 of the GA CR, by Grant S3086102 of GA CAS, and by Projects 1ET400300415 and ME641 of the Ministry of Education of the Czech Republic. The third author was supported by the Research Foundation of the City University of New York Awards PSC-CUNY 665463-00 34 and 66529-00 35. 


\section{References}

1. Avery, P., Rebel, G., Lesoinne, M., Farhat, C.: A numerically scalable Dual-Primal substructuring method for the solution of contact problems - part I: the frictionless case. Computer Methods in Applied Mechanics and Engineering, 193, 2403-2426 (2004)

2. Bertsekas, D. P.: Nonlinear Optimization, Athena Scientific, Belmont (1999).

3. Dostál, Z.: Box constrained quadratic programming with proportioning and projections. SIAM Journal on Optimization 7, 3, 871-887 (1997)

4. Dostál, Z.: A proportioning based algorithm for bound constrained quadratic programming with the rate of convergence. Numerical Algorithms, 34, 2-4, 293-302 (2003)

5. Dostál, Z.: Inexact semi-monotonic Augmented Lagrangians with optimal feasibility convergence for quadratic programming with simple bounds and equality constraints. accepted in SIAM Journal on Numerical Analysis

6. Dostál, Z.: An optimal algorithm for bound and equality constrained quadratic programming problems with bounded spectrum. submitted to Computing

7. Dostál, Z., Friedlander, A., Santos, S.A.: Solution of contact problems of elasticity by FETI domain decomposition. Contemporary Mathematics 218, 82-93 (1998)

8. Dostál, Z., Horák, D., Stefanica, D.: A scalable FETI-DP algorithm for coercive variational inequalities. IMACS Journal Applied Numerical Mathematics, in print

9. Dostál, Z., Horák, D., Stefanica, D.: A Scalable FETI-DP Algorithm with Non-penetration Mortar Conditions on Contact Interface. submitted

10. Dostál, Z., Horák, D., Stefanica, D.: A Scalable FETI-DP Algorithm for Semi-coercive Variational Inequalities. submitted

11. Dostál, Z., Schöberl, J.: Minimizing quadratic functions over non-negative cone with the rate of convergence and finite termination. Computational Optimization and Applications 30, 1, 23-44 (2005)

12. Farhat, C., Lesoinne, M., LeTallec, P., Pierson, K., Rixen, D.: FETI-DP: A dual-prime unified FETI method. I: A faster alternative to the two-level FETI method. International Journal for Numerical Methods Engineering, 50, No.7, 1523-1544 (2001)

13. Farhat, C., Mandel, J., Roux, F.X.: Optimal convergence properties of the FETI domain decomposition method. Computer Methods in Applied Mechanics and Engineering, 115, 365-385 (1994)

14. Farhat, C., Roux, F.X.: An unconventional domain decomposition method for an efficient parallel solution of large-scale finite element systems. SIAM Journal on Scientific Computing 13, 379-396 (1992)

15. Klawonn, A., Widlund, O.B., Dryja, M.: Dual-Primal FETI Methods for Three-dimensional Elliptic Problems with Heterogeneous Coefficients. SIAM Journal on Numerical Analysis, Vol. 40, 159-179 (2002) 